\title{
Polynomial Field Theories and Nonintegrability
}

\author{
N. Euler and W.-H. Steeb \\ Department of Applied Mathematics and Nonlinear Studies, Rand Afrikaans University, PO Box 524 Johannesburg 2000, South Africa \\ and \\ K. Cyrus \\ Department of Computational and Applied Mathematics, University of the Witwatersrand, Johannesburg 2050, South Africa
}

Received June 28, 1989; accepted August 19, 1989

\begin{abstract}
The nonintegrability of the nonlinear field equation $v_{\eta \xi}=v^{3}$ is studied with the help of the Painleve test. The condition at the resonance is discussed in detail. Particluar solutions are given.
\end{abstract}

A remarkable number of exact solutions for nonlinear onedimensional field equations have been found. The nonlinear field equations have been the Korteweg-deVries, nonlinear Schrödinger, sine Gordon, Liouville equation, etc. These equations can be solved exactly within the inverse scattering method or can be transformed to a linear equation (as this is the case for the one-dimensional Liouville equations). All these equations pass the Painleve test. If the Painleve test is passed, then the test can be used to construct Lax pairs and Bäcklund transformations (see Refs [1-5] and references therein).

The formulation is as follows: Assume we have a nonlinear partial differential equation with analytic coefficients (or a set of $N$ partial differential equations for $N$ functions, denoted collectively by $u$ ). Complexify the functions, so that we have $N$ holomorphic functions $u$ of complex variables. Let $\phi$ be a holomorphic function such that $\phi=0$ is not a characteristic hypersurface for the nonlinear partial differential equation. Take the generalized Laurent series $u=\phi^{-m} \sum_{n=0}^{\infty} u_{n} \phi^{n}$ with $m$ being a suitable positive integer, and insert it into the nonlinear partial differential equation. This gives a recursion relation between the expansion coefficients $u_{n}$ which are holomorphic functions of the independent variables. That the nonlinear partial differential equation passes the Painleve test states that these recursion relations should be consistent, and that the generalized Laurent expansion contains the maximal number of arbitrary functions (counting $\phi$ as one of them). This means, in keeping with the Cauchy-Kowaleski theorem such as an expansion of the general solution must have as many arbitrary functions (which are certain of the $u_{n}$ ) as the order of the system. One first determines all possible leading orders (i.e. $m$ and $u_{0}$ ) and then for each leading order one determines the resonances, i.e. the order of $\phi^{n}$ at which the corresponding $u_{n}$ should be arbitrary. The arbitrariness must then be checked by solving the recursion for the $u_{m}$ to ensure that validity of the generalized Laurent expansion. If this is not the case the nonlinear partial differential equation does not pass the Painleve test.

It is well known that in one and more space dimensions polynomial field equations such as the nonlinear Klein-
Gordon equation

$\square u+m^{2} u+\lambda u^{3}=0$

cannot be solved exactly even not for the case $m=0$. Here $\square:=\Delta-\partial^{2} / \partial t^{2}$ and $m$ denotes the mass.

In the present paper we study the nonintegrability of eq. (1) in one space dimension with the help of the Painleve test. For the sake of simplicity we assume that $m=0$, introduce light-cone coordinates $\xi=\frac{1}{2}(x-t), \eta=\frac{1}{2}(x+t)$ and put $\lambda=1$. Then we arrive at

$v_{\eta \xi}=v^{3}$.

It is well known that eq. (1) [and therefore eq. (2)] can be derived from a Lagrangian density and Hamiltonian density [6]. As mentioned above eq. (2) is considered in the complex domain. For the sake of simplicity we do not change our notation.

We focus our attention on three points. First we investigate whether eq. (2) pass the Painlevé test. In particular we give the condition for the singular manifold at the resonance (see Ref. [1] and references therein). This means we insert the expansion

$v=\sum_{j=0}^{\infty} v_{j} \phi^{i-1}$

where $\phi$ and $v_{j}$ are locally holomorphic functions of $\eta$ and $\xi$. For the expansion to be well defined about the manifold

$\phi(\eta, \xi)=0$

it is required that eq. (4) be noncharacteristic for the eq.(2). The resonances are given by $r_{1}=-1$ and $r_{2}=4$. The Kowalewski exponents (see Ref. [1] and references therein) are the same. The Kowalewski exponent $r_{2}=4$ is related to the Hamiltonian density [6]. Second we give the Lie symmetry vector fields of eq. (2) construct the similarity ansatz via the similarity variable $s$ and perform group theoretical reduction of the partial differential equation (2) to ordinary differential equations. The connection of the similarity variables $s$ with the condition on $\phi$ at the resonance is discussed for each of the group theoretical reductions. Furthermore, the Painlevé test is performed for these ordinary differential equations. Finally we discuss the truncated expansion

$v=\phi^{-1} v_{0}+v_{1}$

where $v_{1}$ satisfies eq. (2). Thus the truncated expansion (5) can 
be considered as an auto "Bäcklund transformation", where $\phi$ satisfies certain conditions. This condition, i.e. the partial differential equation for $\phi$, is compared with the condition for $\phi$ at the resonance.

Inserting expansion (3) into eq. (2) gives for the first three expansion "coefficients"

$$
\begin{aligned}
v_{0}^{2} & =2 \phi_{\eta} \phi_{\xi} \\
-\phi_{\xi \eta} v_{0}-\phi_{\xi} v_{0 \eta}-\phi_{\eta} v_{0 \xi} & =3 v_{0}^{2} v_{1} \\
v_{0 \xi \eta} & =3 v_{0}^{2} v_{2}+3 v_{0} v_{1}^{2} \\
2 \phi_{\xi} \phi_{\eta} v_{3}+\phi_{\xi \eta} v_{2}+\phi_{\xi} v_{2 \eta} & +\phi_{\eta} v_{2 \xi}+v_{1 \xi \eta} \\
=3 v_{0}^{2} v_{3}+6 v_{0} v_{1} v_{2} . &
\end{aligned}
$$

At the resonance $r=4$ we obtain

$2 \phi_{\xi \eta} v_{3}+2 \phi_{\eta} v_{3 \xi}+2 \phi_{\xi} v_{3 \eta}+v_{2 \eta \xi}=6 v_{0} v_{1} v_{2} v_{3}+3 v_{0} v_{2}^{2}$.

Inserting system (6) into eq. (7) yields the following condition on $\phi$

$$
\begin{aligned}
3 \phi_{\xi}^{2} \phi_{\eta}^{2} & \left(-\phi_{\xi \xi \xi \xi} \phi_{\eta}^{4}-\phi_{\eta \eta \eta} \phi_{\xi}^{4}+4 \phi_{\xi \eta \eta} \phi_{\xi}^{3} \phi_{\eta}+4 \phi_{\xi \xi \xi \eta} \phi_{\xi} \phi_{\eta}^{3}\right. \\
& \left.+6 \phi_{\xi \xi \eta \eta} \phi_{\xi}^{2} \phi_{\eta}^{2}\right)\left(2 \phi_{\xi \eta} \phi_{\xi} \phi_{\eta}-\phi_{\xi \xi} \phi_{\eta}^{2}-\phi_{\eta \eta} \phi_{\xi}^{2}\right) \\
& -27 \phi_{\xi}^{4} \phi_{\eta}^{4}\left(2 \phi_{\xi \eta \eta} \phi_{\xi \xi \eta} \phi_{\xi} \phi_{\eta}-\phi_{\xi \eta \eta}^{2} \phi_{\xi}^{2}-\phi_{\xi \xi \eta}^{2} \phi_{\eta}^{2}\right) \\
& +66 \phi_{\xi \eta} \phi_{\xi}^{2} \phi_{\eta}^{2}\left(\phi_{\xi \xi \xi} \phi_{\xi \xi} \phi_{\eta}^{5}+\phi_{\eta \eta \eta} \phi_{\eta \eta} \phi_{\xi}^{5}\right) \\
& +6 \phi_{\xi \eta} \phi_{\xi}^{4} \phi_{\eta}^{4}\left(\phi_{\xi \xi \xi} \phi_{\eta \eta} \phi_{\eta}+\phi_{\eta \eta \eta} \phi_{\xi \xi} \phi_{\xi}\right) \\
& -126 \phi_{\xi \eta} \phi_{\xi}^{3} \phi_{\eta}^{3}\left(\phi_{\xi \eta \eta} \phi_{\eta \eta} \phi_{\xi}^{3}+\phi_{\xi \xi \eta} \phi_{\xi \xi} \phi_{\eta}^{3}\right) \\
& -36 \phi_{\eta}^{3} \phi_{\xi}^{3}\left(\phi_{\xi \eta \eta} \phi_{\xi \xi}^{2} \phi_{\eta}^{3}+\phi_{\xi \xi \eta} \phi_{\eta \eta}^{2} \phi_{\xi}^{3}\right) \\
& +18 \phi_{\xi}^{4} \phi_{\eta}^{4}\left(\phi_{\xi \eta \eta} \phi_{\xi \xi \xi} \phi_{\eta}^{2}+\phi_{\xi \xi \eta} \phi_{\eta \eta \eta} \phi_{\xi}^{2}\right) \\
& -18 \phi_{\xi}^{4} \phi_{\eta}^{4}\left(\phi_{\xi \eta \eta} \phi_{\eta \eta \eta} \phi_{\xi}^{2}+\phi_{\xi \xi \eta} \phi_{\xi \xi \xi} \phi_{\eta}^{2}\right) \\
& +54 \phi_{\xi \eta} \phi_{\xi}^{4} \phi_{\eta}^{4}\left(\phi_{\xi \eta \eta} \phi_{\xi \xi} \phi_{\eta}+\phi_{\xi \xi \eta} \phi_{\eta \eta} \phi_{\xi}\right) \\
& +54 \phi_{\xi}^{2} \phi_{\eta}^{2}\left(\phi_{\xi \eta \eta} \phi_{\eta \eta}^{2} \phi_{\xi}^{5}+\phi_{\xi \xi \eta} \phi_{\xi \xi}^{2} \phi_{\eta}^{5}\right) \\
& -24 \phi_{\xi} \phi_{\eta}\left(\phi_{\xi \xi \xi} \phi_{\xi \xi}^{2} \phi_{\eta}^{7}+\phi_{\eta \eta \eta} \phi_{\eta \eta}^{2} \phi_{\xi}^{7}\right) \\
& -6 \phi_{\xi}^{4} \phi_{\eta}^{4}\left(\phi_{\xi \xi \xi} \phi_{\eta \eta \eta} \phi_{\eta} \phi_{\xi}+\phi_{\xi \xi}^{2} \phi_{\eta \eta}^{2}\right) \\
& -88 \phi_{\xi \eta} \phi_{\xi} \phi_{\eta}\left(\phi_{\eta \eta}^{3} \phi_{\xi}^{6}+\phi_{\xi \xi}^{3} \phi_{\eta}^{6}\right) \\
& +3 \phi_{\xi}^{2} \phi_{\eta}^{2}\left(\phi_{\xi \xi \xi}^{2} \phi_{\eta}^{6}+\phi_{\eta \eta \eta}^{2} \phi_{\eta}^{6}\right) \\
& +6 \phi_{\xi}^{4} \phi_{\eta}^{4}\left(\phi_{\xi \xi \xi} \phi_{\eta \eta}^{2} \phi_{\xi}+\phi_{\eta \eta \eta} \phi_{\xi \xi}^{2} \phi_{\eta}\right)+20\left(\phi_{\xi \xi}^{4} \phi_{\eta}^{8}+\phi_{\eta \eta}^{4} \phi_{\xi}^{8}\right) \\
& -4 \phi_{\xi}^{3} \phi_{\eta}^{3} \phi_{\xi \eta}\left(3 \phi_{\xi \xi} \phi_{\eta \eta}+7 \phi_{\eta \xi}^{2}\right)\left(\phi_{\xi \xi} \phi_{\eta}^{2}+\phi_{\eta \eta} \phi_{\xi}^{2}\right) \\
& +18 \phi_{\xi}^{4} \phi_{\eta}^{4}\left(\phi_{\xi \xi} \phi_{\eta \eta}+2 \phi_{\xi \eta}^{2}\right)\left(\phi_{\xi \eta \eta} \phi_{\xi}+\phi_{\xi \xi \eta} \phi_{\eta}\right) \\
& -18 \phi_{\xi}^{3} \phi_{\eta}^{3}\left(\phi_{\xi \xi} \phi_{\eta \eta}+2 \phi_{\xi \eta}^{2}\right)\left(\phi_{\xi \xi \xi} \phi_{\eta}^{3}+\phi_{\eta \eta \eta} \phi_{\xi}^{3}\right) \\
& +\phi_{\xi}^{2} \phi_{\eta}^{2}\left(17 \phi_{\xi \xi} \phi_{\eta \eta}+111 \phi_{\xi \eta}^{2}\right)\left(\phi_{\xi \xi}^{2} \phi_{\eta}^{4}+\phi_{\eta \eta}^{2} \phi_{\xi}^{4}\right) \\
& -2 \phi_{\xi \eta}^{2} \phi_{\xi}^{4} \phi_{\eta}^{4}\left(2 \phi_{\xi \eta}^{2}+15 \phi_{\xi \xi} \phi_{\eta \eta}\right)=0 .
\end{aligned}
$$

If condition (8) is satisfied, then the expansion "coefficient $v_{4}(\xi, \eta)$ " is arbitrary. For example, eq. (8) cannot be satisfied when we set $\phi(\eta, \xi)=\eta \xi$. Consequently, we conclude that eq. (2) does not pass the Painlevé test.

If we assume that $\phi_{\xi \eta}=0$ it follows that

$$
\begin{gathered}
3 \phi_{\xi}^{2} \phi_{\eta}^{2}\left(\phi_{\xi \xi \xi \xi} \phi_{\eta}^{4}+\phi_{\eta \eta \eta \eta} \phi_{\xi}^{4}\right)\left(\phi_{\xi \xi} \phi_{\eta}^{2}+\phi_{\eta \eta} \phi_{\xi}^{2}\right) \\
-24 \phi_{\xi} \phi_{\eta}\left(\phi_{\xi \xi \xi} \phi_{\xi \xi}^{2} \phi_{\eta}^{7}+\phi_{\eta \eta \eta} \phi_{\eta \eta}^{2} \phi_{\xi}^{7}\right) \\
-6 \phi_{\xi}^{4} \phi_{\eta}^{4}\left(\phi_{\xi \xi \xi} \phi_{\eta \eta \eta} \phi_{\eta} \phi_{\xi}+\phi_{\xi \xi}^{2} \phi_{\eta \eta}^{2}\right)
\end{gathered}
$$

$$
\begin{aligned}
& +3 \phi_{\xi}^{2} \phi_{\eta}^{2}\left(\phi_{\xi \xi \xi}^{2} \phi_{\eta}^{6}+\phi_{\eta \eta \eta}^{2} \phi_{\xi}^{6}\right) \\
& +6 \phi_{\xi}^{4} \phi_{\eta}^{4}\left(\phi_{\xi \xi \xi} \phi_{\eta \eta}^{2} \phi_{\xi}+\phi_{\eta \eta \eta} \phi_{\xi \xi}^{2} \phi_{\eta}\right) \\
& +20\left(\phi_{\xi \xi}^{4} \phi_{\eta}^{8}+\phi_{\eta \eta}^{4} \phi_{\xi}^{8}\right) \\
& -18 \phi_{\xi \xi} \phi_{\eta \eta} \phi_{\xi}^{3} \phi_{\eta}^{3}\left(\phi_{\xi \xi \xi} \phi_{\eta}^{3}+\phi_{\eta \eta \eta} \phi_{\xi}^{3}\right) \\
& +17 \phi_{\xi \xi} \phi_{\eta \eta} \phi_{\xi}^{2} \phi_{\eta}^{2}\left(\phi_{\xi \xi}^{2} \phi_{\eta}^{4}+\phi_{\eta \eta}^{2} \phi_{\xi}^{4}\right)=0 .
\end{aligned}
$$

We now consider the reduced singularity manifold $\phi(\eta, \xi)=\eta-g(\xi)=0$.

Then $\phi_{\xi \eta}=0$ and $\phi_{\eta \eta}=0$. Equation (8) reduces to a condition on $g(\xi)$ given by

$3\left(g^{\prime}\right)^{2} g^{\prime \prime} g^{(4)}+3\left(g^{\prime}\right)^{2}\left(g^{(3)}\right)^{2}-24 g^{\prime}\left(g^{\prime \prime}\right)^{2} g^{(3)}+20\left(g^{\prime \prime}\right)^{4}=0$,

where $g^{\prime} \equiv \mathrm{d} g / \mathrm{d} \xi$ and $g^{(4)} \equiv \mathrm{d}^{4} g / \mathrm{d} \xi^{4}$. For $A=\left(\ln g^{\prime}\right)^{\prime}$ eq. (11) reduces to

$3 A A^{\prime \prime}+3\left(A^{\prime}\right)^{2}-9 A^{2} A^{\prime}+2 A^{4}=0$.

Let us now perform a Painlevé test for eq. (12). Inserting the ansatz $A(\xi) \propto A_{0} \xi^{n}$ we find $n=-1$ and $A_{0}$ admits two solutions, namely $A_{0}=-3 / 2$ and $A_{0}=-3$. All terms are dominant. Thus eq. (12) admits two branches in the Painlevé analysis. For the branch with $A_{0}=-3 / 2$ the resonances are given by $r_{1}=-1$ and $r_{2}=3 / 2$. Equation (12) admits an expansion of the form

$A(\xi)=\left(\xi-\xi_{1}\right)^{-1} \sum_{j=0}^{\infty} A_{j}\left(\xi-\xi_{1}\right)^{j / 2}$

where at the resonance $r=3 / 2$ the expansion coefficient is arbitrary. For the second branch we find the resonances $r_{1}=-1$ and $r_{2}=-3$. Thus eq. (12) passes the so-called weak Painlevé test (see Ref [1] and references therein). Owing the two branches of eq. (12) and since all terms are dominant in eq. (12) we find two special solutions for eq. (11) given by

$g(\xi)=-2 \xi^{-1 / 2} ; \quad g(\xi)=-\frac{1}{2} \xi^{-2}$

so that

$\phi(\xi, \eta)=\eta+2 \xi^{-1 / 2} ; \quad \phi(\xi, \eta)=\eta+\frac{1}{2} \xi^{-2}$

satisfy conditions (8) and (9).

System (2) admits the Lie symmetry vector fields

$\left\{\frac{\partial}{\partial \xi}, \frac{\partial}{\partial \eta},-\xi \frac{\partial}{\partial \xi}+\eta \frac{\partial}{\partial \eta},-\xi \frac{\partial}{\partial \eta}-\eta \frac{\partial}{\partial \eta}+v \frac{\partial}{\partial v}\right\}$

The first two Lie symmetry vector fields are related to the fact that eq. (2) does not depend explicitly on $\eta$ and $\xi$. The third Lie symmetry vector field is related to the Lorentz transformation and the fourth is related to the scale invariance of eq. (2), i.e. $\eta \rightarrow \varepsilon^{-1} \eta, \xi \rightarrow \varepsilon^{-1} \xi, v \rightarrow \varepsilon v$. No Lie-Bäcklund vector fields can be found for eq. (2). The symmetry generators $\partial / \partial \xi, \partial / \partial \eta$ lead to the similarity ansatz

$v(\xi, \eta)=f(s)$

where the similarity variable, $s$ is given by $s=c_{1} \xi+c_{2} \eta$. $c_{1}$ and $c_{2}$ are constants. Inserting eq. (17) into eq. (2) yields

$\frac{\mathrm{d}^{2} f}{\mathrm{~d} s^{2}}=\frac{1}{c_{1} c_{2}} f^{3}$.

Equation (18) passes the Painleve test. This is in agreement that $\phi(\xi, \eta)=c_{1} \eta+c_{2} \xi$ satisfies eq. (7). Equation (18) can 
be solved in terms of Jacobi elliptic functions. The symmetry generator $-\xi \partial / \partial \xi+\eta \partial / \partial \eta$ leads to the similarity ansatz

$v(\xi, \eta)=f(s)$

where the similarity variable $s$ is given by $s=\eta \xi$. Inserting eq. (19) into eq. (2) yields

$\frac{\mathrm{d}^{2} f}{\mathrm{~d} s^{2}}+\frac{1}{s} \frac{\mathrm{d} f}{\mathrm{~d} s}-\frac{1}{s} f^{3}=0$.

Equation (20) does not pass the Painleve test. This is in agreement that $\phi(\xi, \eta)=\eta \xi$ does not satisfy eq. (8). The symmetry generator $-\xi \partial / \partial \xi-\eta \partial / \partial \eta+v \partial / \partial v$ leads to the similarity ansatz

$v(\xi, \eta)=\frac{1}{\xi} f(s)$

where the similarity variable $s$ is given by $s=\eta / \xi$. Inserting eq. (21) into eq. (2) yields

$\frac{\mathrm{d}^{2} f}{\mathrm{~d} s^{2}}+\frac{2}{s} \frac{\mathrm{d} f}{\mathrm{~d} s}+\frac{1}{s} f^{3}=0$

Equation (22) passes the Painlevé test. This is in agreement that $\phi(\xi, \eta)=\eta / \xi$ satisfies eq. (8). From the Painlevé analysis we find a particular solution to eq. (22)

$f(s)=\frac{\sqrt{-2 s_{1}}}{s-s_{1}}$.

Let us discuss the truncated ansatz (5). Inserting the truncated ansatz into eq. (2) yields

$$
\begin{aligned}
v_{0}^{2} & =2 \phi_{\eta} \phi_{\xi} \\
-\phi_{\xi \eta} v_{0}-\phi_{\xi} v_{0 \eta}-\phi_{\eta} v_{0 \xi} & =3 v_{0}^{2} v_{1} \\
v_{0 \xi \eta} & =3 v_{0} v_{1}^{2} \\
v_{1 \xi \eta} & =v_{1}^{3} .
\end{aligned}
$$

It follows that

$$
-4 \phi_{\xi} \phi_{\eta} \phi_{\eta \xi}-\phi_{\eta}^{2} \phi_{\xi \xi}-\phi_{\xi}^{2} \phi_{\eta \eta}=6 v_{0} v_{1} \phi_{\xi} \phi_{\eta}
$$

and

$$
\begin{aligned}
12 \phi_{\xi}^{2} \phi_{\eta}^{2} v_{1}^{2}= & \phi_{\eta} \phi_{\xi} \phi_{\eta \eta} \phi_{\xi \xi}-\phi_{\eta}^{2} \phi_{\eta \xi} \phi_{\xi \xi}-\phi_{\xi}^{2} \phi_{\eta \xi} \phi_{\eta \eta} \\
& +2 \phi_{\xi} \phi_{\eta}^{2} \phi_{\eta \xi \xi}+2 \phi_{\xi}^{2} \phi_{\eta} \phi_{\eta \eta \xi}+\phi_{\xi} \phi_{\eta} \phi_{\eta \xi}^{2},
\end{aligned}
$$

where $v_{0}$ is given by eq. (24a). We see that the truncated expansion (5) leads to a different condition on $\phi$ compared to condition (8). If $\phi_{\eta \xi}=0$, it follows that

$-\phi_{\eta}^{2} \phi_{\xi \xi}-\phi_{\xi}^{2} \phi_{\eta \eta}=6 v_{0} v_{1} \phi_{\xi} \phi_{\eta}$

and

$12 \phi_{\xi}^{2} \phi_{\eta}^{2} v_{1}^{2}=\phi_{\eta} \phi_{\xi} \phi_{\eta \eta} \phi_{\xi \xi}$.

Here, too, the condition on $\phi$ is different compared to the condition (9). Solutions can be constructed when we insert a solution of eq. (24d). The simplest case is $v_{1}=0$. Then from eqs. (25), (26) and (5) it follows that

$v(\eta, \xi)=\frac{\sqrt{-2 c_{1} c_{2}}}{c_{1} \xi+c_{2} \eta}$

is a special solution to eq. (2). Whether solution (29) can be used to construct another solution with the help of the "Bäcklund transformation" (5) and the conditions (25) and (26) is not obvious since we have to prove that eq. (25) and eq. (26) are compatible.

\section{References}

1. Steeb, W.-H. and Euler, N., Nonlinear Evolution Equations and Painlevé Test. World Scientific Publishing, Singapore (1988).

Weiss, J., J. Math. Phys. 25, 13 (1984).

3. Ward, R. S., Nonlinearity 1, 671 (1988).

4. Tabor, M. and Gibbon, J. D., Physica 18D 180 (1986).

5. Pogrebkov, A. K., Inverse Probl. 5, L7 (1989).

6. Steeb, W.-H. and Louw, J. A., Prog. Theor. Phys. 76, 1177 (1986). 